\title{
Impact of COVID-19 pandemic on the healthcare and psychosocial well-being of patients with inflammatory bowel disease
}

\author{
Anna Theodorou-Kanakaria, Paraskevas Gkolfakis ${ }^{b}$, Georgios Tziatziosa ${ }^{a}$ Lazaros Dimitrios Lazaridisa, \\ Konstantinos Triantafyllou ${ }^{\mathrm{a}}$
}

Medical School, National and Kapodistrian University of Athens, "Attikon" University General Hospital, Athens, Greece; CUB Erasme Hospital, Université Libre de Bruxelles (ULB), Brussels, Belgium

\section{Abstract}

\begin{abstract}
COVID-19 pandemic has resulted in unprecedented disruptions to several aspects of gastroenterology healthcare services worldwide. In particular, patients with inflammatory bowel disease (IBD) represent a sensitive population that must retain access to healthcare services to avoid potential disease exacerbation under the continuous threat of viral infection. Emerging evidence also highlights the severe impact on these patients' mental well-being, leading to a constant cycle of stress/depression and disease activity relapse. In an effort to circumvent these healthcare challenges in a newly-shaped environment, physicians implemented telemedicine consultative care programs as a novel alternative follow-up method highly favored by the patients. The situation is still far from perfect, since a large proportion of patients are lost to follow up and/ or lose adherence to their medication, especially when the exact timeframe or optimal strategy for the post-COVID era remains to be defined. Cancelation of elective endoscopic procedures has led to a significant decline of new IBD diagnoses. This review summarizes the data on the global impact of COVID-19 on IBD patients' healthcare and their psychosocial status.
\end{abstract}

Keywords COVID-19, inflammatory bowel disease, impact, well-being

Ann Gastroenterol 2022; 35 (X): 1-10

\section{Introduction}

Starting from Wuhan city in Hubei province, China, the novel coronavirus SARS-CoV-2 rapidly spread all around the world, leading to a global health crisis, with millions of cases and deaths. During this period, 6242 patients with

${ }^{a}$ Hepatogastroenterology Unit, Second Department of Internal Medicine - Propaedeutic, Research Institute and Diabetes Center, Medical School, National and Kapodistrian University of Athens, "Attikon" University General Hospital, Athens, Greece (Anna Theodorou-Kanakari, Georgios Tziatzios, Lazaros Dimitrios Lazaridis, Konstantinos Triantafyllou); ${ }^{b}$ Department of Gastroenterology, Hepatopancreatology, and Digestive Oncology, CUB Erasme Hospital, Université Libre de Bruxelles (ULB), Brussels, Belgium (Paraskevas Gkolfakis)

\section{Conflict of Interest: None}

Correspondence to: Konstantinos Triantafyllou, Associate Professor of Gastroenterology, Hepatogastroenterology Unit, Second Department of Internal Medicine - Propaedeutic Research Institute and Diabetes Center, Medical School, National and Kapodistrian University of Athens, "Attikon" University General Hospital, 1, Rimini Street, 12462 Athens, Greece, e-mail: ktriant@med.uoa.gr

Received 8 November 2021; accepted 11 January 2022; published online 10 February 2022

DOI: https://doi.org/10.20524/aog.2022.0686 inflammatory bowel disease (IBD) have been entered into the SECURE-IBD database (until 8 June 2021), of whom 15\% needed hospitalization and $2 \%$ lost their lives [1]. The IBD community worldwide had to face up to these unprecedented circumstances, while maintaining the quality of IBD care standards [2]. Telemedicine replaced face-to-face visits, elective endoscopy and surgical procedures were temporarily postponed, and strict hospital protocols were adopted in an effort to interrupt virus transmission [2,3].

There is unanimity among the International Organization for the Study of Inflammatory Bowel Diseases (IOIBD) [4], the European Crohn's and Colitis Organization (ECCO) [2], the American Gastroenterology Association (AGA) [5] and the Hellenic Study Group for Inflammatory Bowel Diseases (EOMIFNE) [6] that IBD patients are not facing a higher risk of SARS-CoV-2 infection compared to the general population. On the contrary, some might even develop less severe forms when infected [7]. Initially, evidence suggested a greater probability of hospitalization among patients receiving prednisolone, azathioprine, infliximab or adalimumab, compared to other IBD patients [6]. However, negative COVID-19 outcomes have only been associated with active IBD, older age, presence of comorbidities and high doses of corticosteroids, but not with other IBD treatments [8]. Therefore, patients should remain adherent to their medication to avoid an IBD flare that may require steroid therapy or hospitalization, both of which are 
related to a higher risk of an unfavorable COVID-19 outcome compared to the known risks of IBD therapies [5].

All IBD patients are strongly recommended to get vaccinated against SARS-CoV-2 as soon as possible [9]. All available vaccines are considered equally safe and effective for IBD patients; however, vaccine efficacy may be decreased in those receiving systemic corticosteroids. As far as patients under biological treatment are concerned, the only recommendation is to avoid receiving the vaccine on the same day as an infusion/ subcutaneous dose, to avoid misinterpretation in case an adverse event occurs [10].

IBD is a chronic disease requiring tight control of activity, close monitoring of treatment, and a patient-oriented approach focusing on their physical and psychological well-being. Compared to non-IBD peers, they have a greater likelihood of developing depression and anxiety disorders [11], especially in the case of active disease [12]. The COVID-19 pandemic has exacerbated the problem, since individuals in self-isolation or under quarantine measures are more likely to develop mental health problems such as depression and anxiety [13]. This review presents the available evidence about the impact of the COVID-19 pandemic on IBD patients' healthcare and their psychosocial well-being.

\section{Methodology}

The Medline database was searched for eligible studies up to 12 May 2021, using the MeSH terms: ((2019 Novel Coronavirus Disease) OR (2019 Novel Coronavirus Infection) OR (2019nCoV Disease) OR (2019-nCoV Infection) OR (COVID-19 Pandemic) OR (COVID-19 Virus Disease) OR (COVID-19 Virus Infection) OR (COVID19) OR (Coronavirus Disease 2019) OR (Coronavirus Disease-19) OR (SARS Coronavirus 2 Infection) OR (SARS-CoV-2 Infection)) AND ((Inflammatory Bowel Disease) OR (Crohn's Disease) OR (Crohn's Enteritis) OR (Ulcerative Colitis)). All types of studies published in the English language, referring to humans and providing numerical data, were considered eligible for inclusion, while studies in any other language, non-human, ex vivo or pilot studies, editorials, narrative or systematic reviews and case reports/series were excluded from this review. After the exclusion of duplicates and articles deemed irrelevant to the study's aim, 24 original studies are discussed [14-37]. Their characteristics are summarized in Table 1.

\section{Impact of COVID-19 on IBD healthcare}

During the COVID pandemic all IBD units had to remodel their everyday clinical practice, affecting the quality of healthcare services provided to their patients. Guidelines were immediately developed by ECCO [2], BSG [3], IOIBD [4], AGA [5] and EOMIFNE [6] to provide actual guidance regarding the management of IBD patients during the COVID pandemic. However, findings from surveys around the world reveal significant diversity. Most of them were conducted via electronically distributed questionnaires, aiming to evaluate various outcomes such as disease activity, presence of symptoms, adherence to medication, psychological condition, possible worries of the patient, and familiarization with telemedicine.

For the purposes of this review, the impact of the COVID-19 pandemic on IBD patients' healthcare is presented in terms of direct and indirect outcomes. Direct outcomes include flareups, hospitalizations and emergent surgeries, while indirect outcomes include treatment discontinuation, delay in infusion of biological agents, and loss of patient follow up, namely outcomes that could lead to IBD exacerbation and need for intervention.

As presented in Table 2, there is significant variance among the direct outcomes reported across different surveys. Flareup rates fluctuate between 0.5 and 56\%, emergent surgeries between 0.2 and $17.9 \%$, and hospitalization rates between 0.78 and $15.8 \%$. That is also the case for indirect outcomes, where treatment discontinuation varies between 3.73 and 28\%, and delay of infusions from 1.3-69.6\%. The main reason for these outcomes is patients' choice to stop their treatment or delay their infusion due to fear of COVID-19 contamination, especially in healthcare settings. Moreover, 1 of 4 patients have been lost to follow up (range 4-38.7\%).

An observational study performed in Spain aimed to evaluate the impact of COVID-19 on an IBD unit's activity, comparing data to those from the previous year [20]. Sixty-four (76.19\%) of the elective endoscopies and all scheduled surgeries were suspended. This led $9.4 \%$ of patients to experience a flare of IBD symptoms in the first 2 months of lockdown, compared to $6.9 \%$ the previous year ( $2.5 \%$ increase, $\mathrm{P}=0.18$ ). Loss of follow up increased from $3.6 \%$ to $5.1 \%$, and adherence to medication decreased from $99.5 \%$ to $94.9 \%(\mathrm{P}<0.05)$. In particular, the discontinuation of biological treatment was almost 8-fold (from $0.5 \%$ to $3.73 \%, \mathrm{P}<0.05$ ).

As expected, patients who discontinued medication on their own initiative faced an increased risk for adverse outcomes. In a study from China, where guidance through telemedicine was provided, $107 / 386(28 \%)$ of the patients reported treatment discontinuation and 108/386 (28\%) experienced a flare-up [15]. Among those stopping their treatment, 57\% $(61 / 107)$ eventually required hospitalization and $4.7 \%$ (5/107) needed surgical treatment. Hospitalization rates were clearly and significantly higher among patients who discontinued their medication (57\% vs. $0.78-15.8 \%$ ).

It is noteworthy that adherence to the different categories of medication may be variable. An online survey from the United Kingdom [21] showed that the most frequently discontinued medications were oral steroids (43.9\%). Immunomodulators were discontinued by $11.2 \%$ of the patients, 1 of 3 against a physician's recommendation. Contrariwise, adherence to biological agents remained high, since $99 \%$ of them continued their use. Between March and August 2020, 36.6\% reported a flare of IBD symptoms. Almost half of them contacted neither their general practitioner nor the IBD helpline and self-managed their symptoms.

A retrospective study conducted in the Netherlands used the nationwide registry of histo- and cytopathology researchers to 
Table 1 Characteristics of included studies

\begin{tabular}{|c|c|c|c|c|c|c|}
\hline Study, author [ref] & Country & Study period & $\begin{array}{l}\text { Population, } n \\
\text { (CD/UC) }\end{array}$ & $\begin{array}{l}\text { Population } \\
\text { characteristics }\end{array}$ & Methodology & Main outcomes \\
\hline Yu et al [14] & China & $02 / 2020$ & $102(46 / 56)$ & $\begin{array}{l}\text { M: } 66.7 \% \\
\text { Median age } 34 \text { years }\end{array}$ & $\begin{array}{l}\text { Electronic } \\
\text { survey }\end{array}$ & $\begin{array}{l}5 / 102(4.9 \%) \\
\text { discontinued } \mathrm{Tx} \\
27 / 102(26.5 \%) \\
\uparrow \text { disease } \\
\text { activity }\end{array}$ \\
\hline Chen et al [15] & China & $01-03 / 2020$ & $386(311 / 75)$ & $\begin{array}{l}\text { M: } 70.5 \% \\
\text { Mean age } 32.8 \pm 6.8 \\
\text { years }\end{array}$ & $\begin{array}{l}\text { Patient record, } \\
\text { telephone } \\
\text { follow up and } \\
\text { questionnaire }\end{array}$ & $\begin{array}{l}\text { 107/386 (27.7\%) } \\
\text { discontinued Tx } \\
17 \mathrm{UC} \text { and } \\
91 \mathrm{CD} \text { pts } \\
\text { experienced a } \\
\text { flare }\end{array}$ \\
\hline Lan et al [16] & China & $01-03 / 2020$ & $78(64 / 6)$ & $\begin{array}{l}\text { Patients undergoing } \\
\text { IBD-related } \\
\text { surgeries } \\
61.5 \% \text { elective } \\
\text { surgery, } 12(15.4 \%) \\
\text { emergency } \\
\text { surgery, } \\
18 \text { postponed }\end{array}$ & $\begin{array}{l}\text { Anonymous } \\
\text { online survey }\end{array}$ & $\begin{array}{l}7 / 18(38.9 \%) \\
\uparrow \text { symptoms } \\
2 / 12(11.1 \%) \\
\text { emergency } \\
\text { surgery due } \\
\text { to postponed } \\
\text { surgery }\end{array}$ \\
\hline Tian et al [17] & China & $\begin{array}{l}\text { First wave of } \\
\text { Covid-19 }\end{array}$ & $239(71 / 151)$ & $\begin{array}{l}\text { M: } 54.81 \% \\
66.5 \% \text { 5-ASA, } 2.5 \% \\
\text { corticosteroids } \\
14.2 \% \\
\text { immunosuppression, } \\
17.6 \% \\
\text { biological Tx }\end{array}$ & Questionnaires & $\begin{array}{l}51(21.34 \%) \text { IBD } \\
\text { flare } \\
52(21.76 \%) \\
\text { changed Tx } \\
41 / 239(17 \%) \\
\text { delay infusion }\end{array}$ \\
\hline Chen et al [18] & China & $01-04 / 2020$ & $2277(1639 / 555)$ & M: $61 \%$ & $\begin{array}{l}\text { Anonymous } \\
\text { online } \\
\text { questionnaires }\end{array}$ & $\begin{array}{l}585 / 2277(25.7 \%) \\
\text { had a flare } \\
533 / 2277(23.4 \%) \\
\text { discontinued Tx } \\
791 / 2277 \\
(34.74 \%) \text { loss of } \\
\text { follow up } \\
443 / 2277 \text { (39\%) } \\
\text { income } \downarrow\end{array}$ \\
\hline Bai et al [19] & China & $01-02 / 2020$ & $125(45 / 58)$ & $\begin{array}{l}\text { M: } 61.6 \% \\
\text { Median age } 39 \text { years }\end{array}$ & $\begin{array}{l}\text { Questionnaires } \\
\text { through emails, } \\
\text { mobile } \\
\text { communication } \\
\text { applications } \\
\text { and text } \\
\text { messaging }\end{array}$ & $\begin{array}{l}18 / 125(14.4 \%) \\
\text { exacerbation }\end{array}$ \\
\hline El Hajra et al [20] & Spain & $03-05 / 2020$ & $510(303 / 199)$ & $\begin{array}{l}85.49 \% \text { inactive } \\
\text { patients } \\
9.41 \%) \text { IBD flare }\end{array}$ & $\begin{array}{l}\text { Comparison } \\
\text { between } 2020 \\
\text { and } 2019\end{array}$ & $\begin{array}{l}2020 \text { vs. } 2019 \\
\text { IBD flare: } 9.4 \% \\
\text { vs. } 6.9 \% \\
\text { Loss of follow up: } \\
5.1 \% \text { vs. } 3.6 \% \\
\text { Adherence } \\
\text { to Tx: } 94.9 \% \text { vs. } \\
99.5 \% \\
\text { Biological Tx } \\
\text { discontinuation: } \\
3.73 \% \text { vs. } 0.5 \%\end{array}$ \\
\hline
\end{tabular}

(Contd...) 
Table 1 (Continued)

\begin{tabular}{|c|c|c|c|c|c|c|}
\hline Study, author [ref] & Country & Study period & $\begin{array}{l}\text { Population, } \mathrm{n} \\
\text { (CD/UC) }\end{array}$ & $\begin{array}{l}\text { Population } \\
\text { characteristics }\end{array}$ & Methodology & Main outcomes \\
\hline Harris et al [21] & UK & $03-08 / 2020$ & $685(443 / 221)$ & $\begin{array}{l}\text { M: } 42 \% \\
26.3 \% \\
\text { immunomodulators, } \\
28.5 \% \text { on biological/ } \\
\text { small molecules, } \\
16.5 \% \text { dual } \\
\text { immunosuppression, } \\
6 \% \text { oral steroids }\end{array}$ & $\begin{array}{l}\text { Electronically } \\
\text { distributed } \\
\text { questionnaires }\end{array}$ & $\begin{array}{l}251(36.64 \%) \text { had } \\
\text { a flare } \\
104(15.2 \%) \\
\text { emergent surgery } \\
127(19 \%) \\
\text { cancelled } \\
\text { outpatient } \\
\text { appointment } \\
363 / 685(52.99 \%) \\
\text { negative impact } \\
\text { on psychological } \\
\text { well-being }\end{array}$ \\
\hline Goodday et al [22] & UK, USA & $04-06 / 2020$ & $243 \mathrm{CD}$ & $\begin{array}{l}86 \% \text { United } \\
\text { Kingdom } \\
10 \% \text { United States }\end{array}$ & $\begin{array}{l}\text { Anonymous } \\
\text { distributed } \\
\text { survey through } \\
\text { international } \\
\text { gastroenterology } \\
\text { clinics and } \\
\text { networks }\end{array}$ & $\begin{array}{l}24 \% \text { relative } \uparrow \text { in } \\
\text { active symptoms } \\
\text { compared to } \\
\text { pre-COVID-19 } \\
\text { period }(\mathrm{P}<0.001) \\
118 / 243(48.6 \%) \\
\text { due to stress }\end{array}$ \\
\hline Grunert et al [23] & Germany & $04 / 2020$ & $415(215 / 192)$ & $\begin{array}{l}\text { M: } 45.3 \% \\
46.8 \% \text { biological } \\
\text { agents, 34.5\% 5-ASA, } \\
23.9 \% \text { anti-TNF }\end{array}$ & $\begin{array}{l}\text { Matched ( } 4: 1) \\
\text { anonymous } \\
\text { survey of } 415 \\
\text { IBD patients } \\
\text { and } 116 \text { control } \\
\text { participants }\end{array}$ & $\begin{array}{l}\text { IBD patients } \\
\text { significantly } \\
\text { more affected by } \\
\text { the COVID-19 } \\
\text { pandemic than } \\
\text { non-IBD peers, } \\
\text { but generally } \\
\text { remained } \\
\text { adherent to } \\
\text { medication }\end{array}$ \\
\hline $\begin{array}{l}\text { Foteinogiannopoulou } \\
\text { et al [24] }\end{array}$ & Greece & $03-04 / 2020$ & 78 & 64 infusions & $\begin{array}{l}\text { IBD unit } \\
\text { experience } \\
\text { report }\end{array}$ & $\begin{array}{l}58 / 64(90.6 \%) \\
\text { received their } \\
\text { infusion on time }\end{array}$ \\
\hline Turner et al [25] & $\begin{array}{l}\text { China, } \\
\text { South } \\
\text { Korea, } \\
\text { Portugal }\end{array}$ & $01-03 / 2020$ & 446 & $\begin{array}{l}7 \text { children with } \\
\text { IBD infected with } \\
\text { SARS-CoV-2 }\end{array}$ & $\begin{array}{l}\text { Electronic } \\
\text { reporting } \\
\text { system }\end{array}$ & $\begin{array}{l}\text { Pediatric IBD } \\
\text { patients should } \\
\text { not stop their } \\
\text { medication or } \\
\text { delay infusions }\end{array}$ \\
\hline Viola A et al [26] & Italy & $01-07 / 2020$ & $689(369 / 320)$ & $\begin{array}{l}247 \text { patients IV } \\
\text { biologics, } 217 \text { SC } \\
\text { biologics, } 225 \text { oral Tx }\end{array}$ & $\begin{array}{l}\text { Hospital } \\
\text { records during } \\
\text { lockdown } \\
\text { compared with } \\
\text { the respective } \\
\text { period in } 2019\end{array}$ & $\begin{array}{l}\text { Endoscopies } \downarrow 85 \% \\
\text { compared to } 2019\end{array}$ \\
\hline Rizello et al [27] & Italy & $03-06 / 2020$ & $1158(695 / 463)$ & $\begin{array}{l}\text { M: } 55.6 \% \\
56.7 \% \text { on remission }\end{array}$ & $\begin{array}{l}23 \mathrm{MCQ} \\
\text { anonymous } \\
\text { survey }\end{array}$ & $\begin{array}{l}204(17.6 \%) \uparrow \\
\text { symptoms: } \\
102(8.8 \%) \\
\text { discontinued } \mathrm{Tx} \\
244(21.1 \%) \\
\text { delayed infusions }\end{array}$ \\
\hline Zhang et al [28] & China & $12 / 2019-02 / 2020$ & $505(354 / 126)$ & $\begin{array}{l}84 \text { patients at } \\
\text { consultation } \\
56 \text { patients on } \\
\text { anti-TNF infusions }\end{array}$ & $\begin{array}{l}\text { Observational } \\
\text { study on } \\
\text { healthcare and } \\
\text { medication use } \\
\text { Electronic } \\
\text { questionnaire } \\
\text { survey about } \\
\text { telemedicine } \\
\text { acceptance }\end{array}$ & $\begin{array}{l}39 / 56(69.6 \%) \\
\text { delayed infusion } \\
\text { Physicians using } \\
\text { telemedicine } \uparrow \text { by } \\
18.9 \% \text { compared } \\
\text { with the pre- } \\
\text { outbreak period } \\
(\mathrm{p}<0.001)\end{array}$ \\
\hline
\end{tabular}


Table 1 (Continued)

\begin{tabular}{|c|c|c|c|c|c|c|}
\hline Study, author [ref] & Country & Study period & $\begin{array}{l}\text { Population, n } \\
\text { (CD/UC) }\end{array}$ & $\begin{array}{l}\text { Population } \\
\text { characteristics }\end{array}$ & Methodology & Main outcomes \\
\hline Saibeni et al [29] & Italy & $\begin{array}{c}\text { 04/2020 and } \\
06 / 2020\end{array}$ & NR & $\begin{array}{l}52.4 \% \text { in northern } \\
\text { Italy } \\
85.7 \% \text { in public } \\
\text { hospitals, } 54.7 \% \text { in } \\
\text { academic hospitals }\end{array}$ & $\begin{array}{l}\text { Web-based } \\
\text { online survey }\end{array}$ & $\begin{array}{l}>70 \% \text { of follow } \\
\text { up and } 5 \% \text { of } \\
\text { first visits by } \\
\text { virtual clinics } \\
14 \% \text { delay in } \\
\text { infusions }>70 \% \\
\text { of endoscopies } \\
\text { cancelled }\end{array}$ \\
\hline Scaldaferri et al [30] & Italy & $03-04 / 2020$ & $1451(784 / 522)$ & $\begin{array}{l}\text { M: } 58 \% \\
\text { Mean age } 44 \text { years } \\
27 \% \text { infliximab, } 31 \% \\
\text { adalimumab }\end{array}$ & $\begin{array}{l}\text { Observational } \\
\text { prospective } \\
\text { study }\end{array}$ & $\begin{array}{l}222 / 1451 \\
(15.33 \%) \text { delayed } \\
\text { infusions due to } \\
\text { patients' choice }\end{array}$ \\
\hline Costantino et al [31] & Italy & NR & $100(75 / 25)$ & $\begin{array}{l}\text { M: 69\% } \\
\text { Mean age } 41 \text { years }\end{array}$ & $\begin{array}{l}\text { Questionnaire } \\
\text { about patients' } \\
\text { trust in } \\
\text { telemedicine }\end{array}$ & $\begin{array}{l}95 \% \text { of } \\
\text { patients using } \\
\text { telemedicine } \\
\text { trust this method } \\
\text { of follow up }\end{array}$ \\
\hline Allocca et al [32] & Italy & $\begin{array}{l}\text { First wave of } \\
\text { COVID-19 }\end{array}$ & 725 & $\begin{array}{l}700 \text { patients already } \\
\text { on IV or SC } \\
25 \text { patients started } \\
\text { infusions during the } \\
\text { study }\end{array}$ & $\begin{array}{l}\text { IBD unit } \\
\text { experience } \\
\text { report }\end{array}$ & $\begin{array}{l}350 \% \uparrow \text { of contacts } \\
\text { in helpline after } \\
\text { changes in the } \\
\text { restriction rules }\end{array}$ \\
\hline Iborra et al [33] & Spain & $03-04 / 2020$ & $234(178 / 52)$ & $\begin{array}{l}\text { Database: M: } 53 \% \text {, } \\
117 \text { IV and } 117 \\
\text { SC Tx } \\
\text { Telephone survey: } \\
155 \text { ( } 77 \text { IV, } 78 \text { SC) }\end{array}$ & $\begin{array}{l}\text { Cross-sectional } \\
\text { observational } \\
\text { study }\end{array}$ & $\begin{array}{l}\text { Database: } 12 / 117 \\
(10.26 \%) \text { and } \\
5 / 117(5.13 \%) \\
\text { delayed IV and } \\
\text { SC agents } \\
\text { Phone survey: } \\
26 / 155 \text { (17\%) } \\
\text { postponed Tx } \\
\text { (50\% fear of going } \\
\text { to the hospital) }\end{array}$ \\
\hline Clough et al [34] & UK & $05 / 2020$ & 107 & $\begin{array}{l}55.7 \% \text { of patients } \\
\text { attended infusion } \\
\text { during survey period }\end{array}$ & Questionnaire & $\begin{array}{l}80.2 \% \text { consider } \\
\text { IBD unit's } \\
\text { measures to } \\
\text { prevent COVID } \\
\text { to be adequate }\end{array}$ \\
\hline Schlabitz et al [35] & Germany & $04-07 / 2020$ & 1199 & $\begin{array}{l}\text { M: } 23 \\
\text { Mean age } 41.3 \text { years }\end{array}$ & $\begin{array}{l}40 \text {-item } \\
\text { web-based } \\
\text { questionnaire } \\
\text { on } \\
\text { disease-related } \\
\text { experiences and } \\
\text { concerns }\end{array}$ & $\begin{array}{l}38.7 \% \text { of } \\
\text { appointments } \\
\text { cancelled } \\
6.9 \% \mathrm{Tx} \\
\text { discontinuation } \\
71 \% \text { considered } \\
\text { telemedicine as } \\
\text { acceptable }\end{array}$ \\
\hline Trindade et al [36] & Portugal & $04 / 2020$ & 124 & $\begin{array}{l}\text { M: } 14.52 \% \\
\text { Mean age } 40 \text { years } \\
75.8 \% \\
\text { immunosuppressants, } \\
\text { biologics or } \\
\text { corticosteroids }\end{array}$ & $\begin{array}{l}\text { Online } \\
\text { questionnaire }\end{array}$ & $\begin{array}{l}87 / 124(70.1 \%) \\
\text { reported } \\
\text { anxiety and } \\
60 / 124(48.3 \%) \\
\text { depression } \\
\text { symptoms }\end{array}$ \\
\hline Feitosa et al [37] & Brazil & $05 / 2020$ & $570(179 / 46)$ & $\begin{array}{l}\text { M: } 53.3 \% \\
\text { Mean age } 41.3 \text { years }\end{array}$ & $\begin{array}{l}\text { Cross-sectional } \\
\text { study by } \\
\text { personalized } \\
\text { questionnaire }\end{array}$ & $\begin{array}{l}64.9 \% \\
\text { self-quarantined } \\
80.2 \% \text { depression, } \\
58.2 \% \text { anxiety/ } \\
\text { fear of death }\end{array}$ \\
\hline
\end{tabular}


Table 2 Effects of COVID-19 pandemic on IBD patients' healthcare

\begin{tabular}{|c|c|c|c|c|c|c|c|}
\hline \multirow[t]{2}{*}{ Study Author [ref] } & \multicolumn{3}{|c|}{ Direct outcomes, n/N (\%) } & \multicolumn{4}{|c|}{ Indirect outcomes } \\
\hline & Flare-up & $\begin{array}{l}\text { Emergent } \\
\text { surgery }\end{array}$ & Hospitalization & $\begin{array}{c}\text { Treatment } \\
\text { discontinuation, } \\
\mathrm{n} / \mathrm{N}(\%)\end{array}$ & $\begin{array}{c}\text { Reason for } \\
\text { discontinuation }\end{array}$ & $\begin{array}{c}\text { Delayed } \\
\text { infusions, } \\
\mathrm{n} / \mathrm{N}(\%)\end{array}$ & $\begin{array}{l}\text { Loss of } \\
\text { follow up, } \\
\mathrm{n} / \mathrm{N}(\%)\end{array}$ \\
\hline Yu et al [14] & $27 / 102(26.5)$ & NR & NR & $5 / 102(4.9)$ & medication UA & NR & NR \\
\hline Chen et al [15] & $108 / 386(28)$ & $\begin{array}{c}5 / 386 \\
(1.3)\end{array}$ & $61 / 386(15.8)$ & $107 / 386(28)$ & $\begin{array}{l}87 \% \text { traffic } \\
\text { restrictions } \\
13 \% \text { PC }\end{array}$ & NR & NR \\
\hline Lan et al [16] & NR & $\begin{array}{c}14 / 78 \\
(17.95)\end{array}$ & NR & NR & NR & NR & NR \\
\hline Tian et al [17] & $51 / 239(21.3)$ & NR & NR & $61 / 239(25.5)$ & $\begin{array}{c}16.4 \% \\
\text { medication UA } \\
16.4 \% \text { PC }\end{array}$ & $41 / 239(17)$ & NR \\
\hline Chen et al [18] & $\begin{array}{c}585 / 2277 \\
(25.7)\end{array}$ & $\begin{array}{c}23 / 2277 \\
(1)\end{array}$ & $137 / 2277(6)$ & $533 / 2277(23.4)$ & $\begin{array}{c}28 \% \\
\text { physicians/ } \\
\text { facilities UA }\end{array}$ & $\begin{array}{l}\text { 151/533 (28) } \\
\text { infusion UA }\end{array}$ & $\begin{array}{c}791 / 2277 \\
(34.74)\end{array}$ \\
\hline Bai et al [19] & $18 / 125(14.4)$ & NR & NR & NR & NR & NR & $\begin{array}{l}25 / 150 \\
(16.67)\end{array}$ \\
\hline El Hajra et al [20] & $48 / 510(9.4)$ & $\begin{array}{l}1 / 510 \\
(0.2)\end{array}$ & $12 / 510(2.35)$ & $\begin{array}{c}26 / 510(5.1) \\
19 / 510(3.73) \\
\text { biological } \\
\text { agents }\end{array}$ & $73.08 \%$ PC & NR & $\begin{array}{c}26 / 510 \\
(5.1)\end{array}$ \\
\hline Harris et al [21] & $\begin{array}{l}251 / 685 \\
(36.64)\end{array}$ & NR & NR & $104 / 685(15.18)$ & $26 \% \mathrm{PC}$ & $18 / 629(2.9)$ & $\begin{array}{l}2015 / 2700 \\
(74.63)\end{array}$ \\
\hline Goodday et al [22] & $136 / 243(56)$ & NR & NR & NR & NR & NR & NR \\
\hline Grunert et al [23] & $21 / 415(0.5)$ & NR & NR & $15 / 415(3.6)$ & PC & NR & NR \\
\hline $\begin{array}{l}\text { Foteinogiannopoulou } \\
\text { et al [24] }\end{array}$ & $4 / 67(5.97)$ & NR & $10 / 67(14.92)$ & $3 / 67(3.4)$ & PC & $4 / 64(6.3)$ & $\begin{array}{l}11 / 78 \\
(14.1)\end{array}$ \\
\hline Turner et al [25] & $\begin{array}{c}\text { 14/66 (21.2) } \\
\text { China } \\
3 / 13(23) \\
\text { Korea }\end{array}$ & NR & $\begin{array}{l}\text { 10/66 (15.2) } \\
\text { China }\end{array}$ & $\begin{array}{l}\text { 2/233 (0.9) } \\
\text { China }\end{array}$ & PC & $\begin{array}{c}\text { 66/233 (28) } \\
\text { China } \\
\text { 13/272 (4.8) } \\
\text { Korea }\end{array}$ & NR \\
\hline Viola A et al [26] & $28 / 689(4.06)$ & NR & $6 / 689(0.87)$ & $45 / 689(6.53)$ & PC & $38 / 247(15)$ & NR \\
\hline Rizello et al [27] & $\begin{array}{c}204 / 1158 \\
(17.6)\end{array}$ & NR & $9 / 1158(0.78)$ & $102 / 1158(8.8)$ & PC & $\begin{array}{l}244 / 1158 \\
(21.1)\end{array}$ & NR \\
\hline Zhang et al [28] & $\begin{array}{c}14 / 84(16.7) \\
5 / 39(12.8)\end{array}$ & $\begin{array}{c}4 / 84 \\
(4.76)\end{array}$ & $7 / 84(8.33)$ & NR & NR & $39 / 56(69.6)$ & NR \\
\hline Saibeni et al [29] & NR & NR & NR & NR & NR & $\begin{array}{l}\sim 663 / 4733 \\
\quad(14)\end{array}$ & $\begin{array}{l}\sim 800 / 3984 \\
\quad(20)\end{array}$ \\
\hline Scaldaferri et al [30] & NR & NR & NR & NR & $15.33 \% \mathrm{PC}$ & $\begin{array}{c}233 / 1451 \\
(16.06)\end{array}$ & NR \\
\hline Allocca et al [32] & NR & NR & NR & NR & NR & $\begin{array}{c}9 / 700(1.3) \\
3 / 25(12) \\
\text { of new } \\
\text { infusions }\end{array}$ & $\begin{array}{c}1 / 25(4) \\
\text { of new } \\
\text { infusions }\end{array}$ \\
\hline Iborra et al [33] & NR & NR & NR & NR & PC & $\begin{array}{c}12 / 117 \\
(10.26) \mathrm{IV} \\
6 / 117(5.13) \\
\text { SC agents }\end{array}$ & NR \\
\hline Clough et al [34] & NR & NR & NR & 20/212 (9.4) & NR & NR & NR \\
\hline
\end{tabular}


Table 2 (Continued)

\begin{tabular}{|c|c|c|c|c|c|c|c|}
\hline \multirow[t]{2}{*}{ Study Author [ref] } & \multicolumn{3}{|c|}{ Direct outcomes, n/N (\%) } & \multicolumn{4}{|c|}{ Indirect outcomes } \\
\hline & Flare-up & $\begin{array}{c}\text { Emergent } \\
\text { surgery }\end{array}$ & Hospitalization & $\begin{array}{c}\text { Treatment } \\
\text { discontinuation, } \\
\mathrm{n} / \mathrm{N}(\%)\end{array}$ & $\begin{array}{c}\text { Reason for } \\
\text { discontinuation }\end{array}$ & $\begin{array}{c}\text { Delayed } \\
\text { infusions, } \\
\text { n/N (\%) }\end{array}$ & $\begin{array}{l}\text { Loss of } \\
\text { follow up, } \\
\mathrm{n} / \mathrm{N}(\%)\end{array}$ \\
\hline Schlabitz et al [35] & NR & NR & NR & $83 / 1199(6.9)$ & NR & NR & $\begin{array}{c}464 / 1199 \\
(38.7)\end{array}$ \\
\hline Trindade et al [36] & NR & NR & NR & $5 / 124(4.03)$ & PC & NR & NR \\
\hline Feitosa et al [37] & NR & NR & NR & $64 / 225(28.4)$ & PC & NR & NR \\
\hline
\end{tabular}

NR, not reported, $P C$, patient choice, UA, unavailability, IV, intravenous, SC, subcutaneous

compare the number of IBD-related procedures between 2020 and 2019 [38]. Overall, 14.2\% fewer IBD-related procedures were performed between February and August 2020. More specifically, at the peak of the pandemic (April) the decline was almost $60 \%$, while 125 fewer new diagnoses and 214 fewer lowgrade dysplasia diagnoses were established, corresponding to reductions of $6.5 \%$ and $25.5 \%$, respectively, compared to the previous year. Notably, no decline in high grade dysplasia or colorectal cancer was recorded.

Apart from the direct and indirect outcomes mentioned above, all aspects of IBD patients' healthcare were also disrupted. A questionnaire survey conducted by phone in Brazil to estimate the impact of the pandemic on IBD follow up and on patients' well-being [37] showed that medical therapy was discontinued by $28.4 \%$ of the patients, with fear of infection being the main reason. In addition, $83 \%$ missed at least one medical appointment, $45.5 \%$ missed their laboratory test, while $17 \%$ did not have any access to medical prescriptions or skipped their endoscopy.

\section{Impact of COVID-19 on psychosocial well-being of IBD patients}

There are many surveys featuring the negative impact of lockdown combined with the fear of SARS-CoV-2 infection on the psychosocial well-being of patients with IBD. For the purposes of this review, psychological outcomes have been divided into reported "negative mood", depression and anxiety (Table 3). A point that deserves attention is the fact that, in the majority of the studies $(n=5 / 6)$, establishment of a mood disorder was not achieved via implementation of a validated questionnaire, but based on a simple report by the patient evaluated through scales created by the authors themselves; thus, all results should be interpreted cautiously. Only the study by Trindale et al [36] used a validated instrument, the Hospital Anxiety and Depression Scale (HADS), specifically designed to address anxiety and depression as 2 separate subscales. Similarly, the validated Short Inflammatory Bowel Disease Questionnaire (SIBDQ) was used to assess the significant aspect of Health-Related Quality of Life (HRQoL) in the study by Yu et al [14]. As shown in Table 3, reports about "negative mood" varied between 0.5 and $52.99 \%$, while regarding depression and anxiety the respective percentages ranged from $52.4-58.2 \%$ and $2.98-80 \%$. Stress and depression may lead to IBD exacerbation, while flares can be followed by depression and anxiety, leading to a self-perpetuating cycle [12]. This is corroborated by a survey from the UK, focusing on stress-related consequences of the pandemic in patients with Crohn's disease [22]. A total of 136 (56\%) patients reported symptoms of disease activity. There was a $24 \%$ relative increase in active symptoms during the lockdown compared with the previous months. "Increased stress and/ or feeling of being overwhelmed" (118/236) was reported as the cardinal reason for the change. Among those reporting stress at the time of the survey, the relative increase in active symptoms was even more pronounced (42\%). Despite the fact that IBD patients face no greater risk of SARS-CoV-2 infection [2-5], they seem to be more afraid than their nonIBD peers, since $38.2 \%$ of them admitted to leaving the house less frequently than their flat mates [23]. Findings from a similar survey [36] demonstrated that people in isolation had more symptoms of depression, but fewer symptoms of anxiety, because of the low fear of getting infected. According to the same survey, IBD patients reported that the pandemic had a negative or an extremely negative impact on their quality of life $(42.8 \%$ and $10.2 \%$, respectively). This impact was worse among younger adults. Almost half of the patients reported insomnia, reduced daily activity and productivity, as well as sexual dysfunction [37].

Data comparing the psychosocial status of IBD patients between the pre- and during-COVID-19 periods remain scant and heterogeneous. Harris et al [21] documented a significant increase in overall stress between the 2 eras (mean stress score pre-lockdown 4.0 vs. 5.5 during lockdown). Moreover, a history of anxiety or depression correlated with greater stress scores pre-lockdown $(\mathrm{P}=0.0005)$, during lockdown $(\mathrm{P}=0.0005)$ and concerns about a "second wave" $(\mathrm{P}=0.008)$. In another study, the proportion of respondents feeling "stressed" of "very stressed" was significantly higher during the COVID-19 period compared to the pre-COVID-19 era ( $n=122 / 236,52 \%$ vs. $n=73 / 236,31 \%$ ) [22]. More importantly, not only were these higher stress levels identified as the reason for a change in symptoms from pre- to during COVID-19, but also the percentage increase in active symptoms was more pronounced among those reporting current stress $(42 \%$, $\mathrm{n}=122, \mathrm{P}<0.0001)$. 
Table 3 Psychological impact of COVID-19 pandemic on IBD patients

\begin{tabular}{|c|c|c|c|}
\hline \multirow[t]{2}{*}{ Study (Author [ref]) } & \multicolumn{3}{|c|}{ Psychosocial impact } \\
\hline & Negative mood, n/N (\%) & Depression, $\mathrm{n} / \mathrm{N}(\%)$ & Anxiety, n/N (\%) \\
\hline Yu et al $[14]$ & $7 / 102(6.86)$ & NR & NR \\
\hline Harris et al [21] & $363 / 685(52.99)$ & NR & NR \\
\hline Goodday et al [22] & $118 / 236(0.5)$ & NR & NR \\
\hline Grunert et al [23] & $\begin{array}{l}159 / 415(38.2) \text { leave house } \\
\text { less frequently than peers }\end{array}$ & NR & NR \\
\hline Foteinogiannopoulou et al [24] & NR & NR & 2/67 (2.98) needed psychiatric medication \\
\hline Trindade et al [36] & NR & $65 / 124(52.41)$ & $62 / 124(50)$ \\
\hline Feitosa et al [37] & NR & $131 / 225(58.2)$ & $180 / 225(80)$ \\
\hline
\end{tabular}

NR, not reported

\section{Emergence of telemedicine}

The doctor-patient relationship has been always the cornerstone of IBD patient healthcare. Clinical visits were the most common manner of follow up. However, the urgent need for telemedicine during the COVID-19 pandemic probably introduced long-term changes in medical practice. Findings of a tertiary referral center for IBD in Italy, which has largely embraced telemedicine, especially video-consultations, suggested that $95 \%$ of their patients using telemedicine trusted this method of follow up [31]. Another survey revealed that, although $65.8 \%$ of patients preferred face-to-face visits during flares, $67.3 \%$ of them preferred telephone follow up at remission in the post-COVID era [21]. Regarding gastrointestinal physicians, the majority $(72.0 \%)$ agree that telemedicine should partly replace clinic visits in the future [28].

\section{Discussion}

The healthcare environment of IBD patients has been greatly affected by the COVID-19 pandemic. Although telemedicine rapidly emerged to replace clinical visits, it cannot fully take their place. Many visits were postponed around the world without being replaced by virtual meetings, especially in the case of first visits, rather than follow ups. The greatest limitation of the studies conducted in this COVID-19 era is that a significant number of patients did not participate in the online surveys. It is possible that people who did not participate in the surveys might have had risky behavior, such as non-adherence to medications [39]. Hence, the actual impact of the pandemic may be even larger in the real world setting, and studies seem to underestimate it. To make things even more ambiguous, one should take into account that the pandemic is not over yet and the optimal strategy for restarting remains unknown. Thus, the consequences will not be fully understood until later. Another limitation is associated with the heterogeneity in methods, aims and patient cohorts among the aforementioned studies. As a result, the data are not easily comparable, warranting further and more detailed studies.
A third limitation concerns the number of deaths due to disease exacerbation, which were excluded from the strict/ direct outcomes, since the studies were conducted mostly via questionnaires and not using hospital medical files. Moreover, reported flare-ups were mostly based on patients' symptoms and were not confirmed by either endoscopy or laboratory findings. Notably, symptoms like diarrhea, even increased fecal calprotectin (FC) levels, have been associated not only with IBD flare but also with COVID-19 disease [40].

Indeed, diarrhea represents a common symptom of COVID-19 infection, and for almost $10 \%$ of non-IBD patients can be the only symptom, without respiratory involvement [41]. These patients are at risk for a severe COVID-19 disease outcome, probably because of the delay in diagnosis. This percentage is even higher among IBD patients, according to a recent systematic review [42].

FC, a stool marker extremely useful in the differential diagnosis of an IBD flare-up, has been suggested as a homebased test for remote monitoring during the pandemic. However, there are indications that FC can be elevated in COVID-19 positive patients, especially among those with symptoms of diarrhea [40]. This is in line with the findings of a systematic review, according to which more than half of SARS-CoV-2 positive patients with gastrointestinal symptoms had elevated FC [43]. This means that the use of FC as a tool during the follow up of IBD patients is problematic during the pandemic, especially in the discrimination between COVID-19 infection and an IBD flare.

Another important issue is postponed endoscopies. Endoscopic procedures are an integral part of IBD surveillance, especially in monitoring therapy efficacy and screening for dysplasia or cancer [44]. Long waiting lists in the postpandemic era will bring the need for specific prioritization. Experts recommend [45] that, during the first 3-6 months after lockdown, priority should be given to mild or moderate flares, subacute bowel obstruction, new IBD diagnosis with abnormal biochemical test, and surveillance for colorectal cancer and postoperative recurrences. All patients should be tested for COVID before the procedure. Capsule endoscopy (CE) could find a breeding ground in the post COVID era [46]. CE could play an important role in Crohn's disease activity monitoring 
before and after the escalation of treatment, as it provides an alternative evaluation method that will offload the pressure from endoscopy departments.

Fear of SARS-CoV-2 infection in healthcare settings is one of the main reasons for non-adherence to medication during the pandemic. However, adherence to biological treatment was already a major issue even before the pandemic. A systematic review in 2013 reported that pooled adherence to anti-tumor necrosis factor (TNF) was $82.6 \%$ (83\% for adalimumab and $71 \%$ for infliximab) [47]. As a result of anti-TNF discontinuation, $55 \%$ of patients with Crohn's disease or ulcerative colitis relapse after 32 and 18 months, respectively [48]. Hence, the effects of treatment discontinuation on patient health during the COVID era will be best evaluated in the near future. On the other hand, delays in the management of IBD patients due to limited access to the healthcare system will also have a huge impact on their overall well-being. A recent study estimated that a 3-month delay in cancer surgery, due to focusing on COVID-19 care, reduces the benefit in life-years gained of all COVID-19 care by $19 \%$ [49].

Hence, in light of the community's uncertainty-despite the vaccination-about when we will fully return to normality, there are some issues that needed to be directly addressed. Firstly, IBD units should actively ensure that patients will not delay their infusions. Phone calls 1 or 2 days earlier than the infusion could play a key role in patient adherence. Moreover, medical staff should be familiar with telemedicine. Home care nurses could play a crucial role in this direction by roughly assessing IBD patients well-being, carrying out blood tests and collecting FC samples. Lastly, effective and frequent communication between doctor and patient is the steppingstone for patients to express their worries or practical problems. Of course, they should be strongly advised to remain adherent to their medication, get vaccinated and apply hygiene measures, but at the same time to exercise, keep up with their hobbies and close friends if possible, so they can preserve their physical and mental well-being. From the clinician's point of view, all appropriate measures that should be implemented to optimize IBD patients' management (assessment according to severity of IBD and concurrent underlying COVID-19 infection, as well as prevention of infection transmission and management of non-COVID IBD patients) are thoroughly summarized within currently available guidelines $[4,5]$. Rather than focusing on efforts to address this topic, our review adds to the existing literature by identifying gaps in current knowledge, discussing important flaws of study design, and contextualizing the benefit of existing studies for everyday clinical practice.

\section{Concluding remarks}

The COVID-19 pandemic has negatively affected the quality of healthcare of IBD patients. Despite the widespread use of telemedicine, a large proportion of patients have been lost to follow up, while others failed to remain adherent to their medication. A pronounced decline in new IBD diagnoses has also been reported. Moreover, the pandemic has had a detrimental psychological impact on IBD patients, leading to a self-perpetuating cycle of stress/depression and disease activity. Telemedicine has been gaining ground as a possible alternative follow-up method in IBD remission, accepted both by patients and physicians.

\section{References}

1. Surveillance Epidemiology of Coronavirus Under Research Exclusion (SECURE-IBD) Database. Available from: https:// covidibd.org/[Accessed 18 January 2022].

2. Fiorino G, Lytras T, Younge L, et al. Quality of care standards in inflammatory bowel diseases: a European Crohn's and Colitis Organisation [ECCO] position paper. J Crohns Colitis 2020;14:1037-1048.

3. Kennedy NA, Jones GR, Lamb CA, et al. British Society of Gastroenterology guidance for management of inflammatory bowel disease during the COVID-19 pandemic. Gut 2020;69:984-990.

4. Rubin DT, Abreu MT, Rai V, Siegel CA; International Organization for the Study of Inflammatory Bowel Disease. Management of patients with Crohn's disease and ulcerative colitis during the coronavirus disease-2019 pandemic: results of an international meeting. Gastroenterology 2020;159:6-13.

5. Rubin DT, Feuerstein JD, Wang AY, Cohen RD. AGA clinical practice update on management of inflammatory bowel disease during the COVID-19 pandemic: expert commentary. Gastroenterology 2020;159:350-357.

6. Apostolou T, Koutroubakis IE, Manolakopoulos S, et al. Management of patients with inflammatory diseases during the COVID-19 pandemic. Mediterr J Rheumatol 2020;31:295-297.

7. Allocca M, Fiorino G, Zallot C, et al. Incidence and patterns of COVID-19 among inflammatory bowel disease patients from the Nancy and Milan cohorts. Clin Gastroenterol Hepatol 2020;18:2134-2135.

8. Brenner EJ, Ungaro RC, Gearry RB, et al. Corticosteroids, but not TNF antagonists, are associated with adverse COVID-19 outcomes in patients with inflammatory bowel diseases: results from an international registry. Gastroenterology 2020;159:481-491.

9. Siegel CA, Melmed GY, McGovern DP, et al; International Organization for the Study of Inflammatory Bowel Diseases (IOIBD). SARS-CoV-2 vaccination for patients with inflammatory bowel diseases: recommendations from an international consensus meeting. Gut 2021;70:635-640.

10. Queiroz NSF, Teixeira FV. Brazilian IBD study group position statement on SARS-COV2 vaccination. Arq Gastroenterol 2021;58:1-4.

11. Lewis K, Marrie RA, Bernstein $\mathrm{CN}$, et al; CIHR team in defining the burden and managing the effects of immunemediated inflammatory disease. The prevalence and risk factors of undiagnosed depression and anxiety disorders among patients with inflammatory bowel disease. Inflamm Bowel Dis 2019;25:1674-1680.

12. Mikocka-Walus A, Knowles SR, Keefer L, Graff L. Controversies revisited: a systematic review of the comorbidity of depression and anxiety with inflammatory bowel diseases. Inflamm Bowel Dis 2016;22:752-762.

13. Brooks SK, Webster RK, Smith LE, et al. The psychological impact of quarantine and how to reduce it: rapid review of the evidence. Lancet 2020;395:912-920.

14. Yu M, Ye Z, Chen Y, et al. Questionnaire assessment helps the selfmanagement of patients with inflammatory bowel disease during the outbreak of coronavirus disease 2019. Aging (Albany NY) 
2020;12:12468-12478.

15. Chen J, Peng $X$, Zhang $M$, Zhi $M$. Impact of medication discontinuation on patients with inflammatory bowel disease during the COVID-19 outbreak. Gastroenterology 2021;160:2223.

16. Lan N, Zhi M, Chen Y, et al. Experience of hospital admission and surgery during the COVID-19 pandemic: a survey of IBD patients. J Gastrointest Surg 2021;25:282-286.

17. Tian WN, Huang YH, Dai C. The effect of the COVID-19 pandemic on the medical mode of patients with inflammatory bowel disease in China. Inflamm Bowel Dis 2020;26:e116-e117.

18. Chen Y, Hu S, Wu H, et al. Patterns of care for inflammatory bowel disease in China during the COVID-19 pandemic. Lancet Gastroenterol Hepatol 2020;5:632-634.

19. Bai X, Yang H, Qian J. COVID-19 outbreak and inflammatory bowel disease management: a questionnaire survey from realistic practice. J Crohns Colitis 2020;14:1494-1495.

20. El Hajra I, Calvo M, Santos Pérez E, et al. Consequences and management of COVID-19 on the care activity of an inflammatory bowel disease unit. Rev Esp Enferm Dig 2021;113:98-102.

21. Harris RJ, Downey L, Smith TR, Cummings JRF, Felwick R, Gwiggner M. Life in lockdown: experiences of patients with IBD during COVID-19. BMJ Open Gastroenterol 2020;7:e000541.

22. Goodday SM, Travis S, Walsh A, Friend SH. Stress-related consequences of the coronavirus disease 2019 pandemic on symptoms of Crohn's disease. Eur J Gastroenterol Hepatol 2021;33:1511-1516.

23. Grunert PC, Reuken PA, Stallhofer J, Teich N, Stallmach A. Inflammatory bowel fisease in the COVID-19 pandemic: the patients' perspective. J Crohns Colitis 2020;14:1702-1708.

24. Foteinogiannopoulou K, Orfanoudaki E, Koutroubakis IE. Keeping on the high quality of health care in Greek inflammatory bowel disease patients in the SARS-CoV-2 era. Clin Gastroenterol Hepatol 2020;18:2380-2381.

25. Turner D, Huang Y, Martín-de-Carpi J, et al; Paediatric IBD Porto group of ESPGHAN. Corona virus disease 2019 and paediatric inflammatory bowel diseases: global experience and provisional guidance (March 2020) from the Paediatric IBD Porto Group of European Society of Paediatric Gastroenterology, Hepatology, and Nutrition. J Pediatr Gastroenterol Nutr 2020;70:727-733.

26. Viola A, Giambò F, Chiappetta MF, Costantino G, Pallio S, Fries W. Management of patients with inflammatory bowel disease and outcomes during the first wave of the Covid-19 pandemic. Dig Liver Dis 2021;53:689-690.

27. Rizzello F, Calabrese C, Salice M, et al. COVID-19 in IBD: The experience of a single tertiary IBD center. Dig Liver Dis 2021;53:271-276.

28. Zhang YF, Qiu Y, He JS, et al. Impact of COVID-19 outbreak on the care of patients with inflammatory bowel disease: A comparison before and after the outbreak in South China. J Gastroenterol Hepatol 2021;36:700-709.

29. Saibeni S, Scucchi L, Dragoni G, et al; IG-IBD (Italian Group for the study of Inflammatory Bowel Disease). Activities related to inflammatory bowel disease management during and after the coronavirus disease 2019 lockdown in Italy: How to maintain standards of care. United European Gastroenterol J 2020;8:1228-1235.

30. Scaldaferri F, Pugliese D, Privitera G, et al. Impact of COVID-19 pandemic on the daily management of biotechnological therapy in inflammatory bowel disease patients: reorganisational response in a high-volume Italian inflammatory bowel disease centre. United European Gastroenterol J 2020;8:775-781.

31. Costantino A, Noviello D, Mazza S, Berté R, Caprioli F, Vecchi M. Trust in telemedicine from IBD outpatients during the COVID-19 pandemic. Dig Liver Dis 2021;53:291-294.

32. Allocca M, Fiorino G, Furfaro F, et al. Maintaining the quality standards of care for inflammatory bowel disease patients during the COVID-19 pandemic. Clin Gastroenterol Hepatol 2020;18:1882-1883.

33. Iborra I, Puig M, Marin L, et al. Treatment adherence and clinical outcomes of patients with inflammatory bowel disease on biological agents during the SARS-CoV-2 pandemic. Dig Dis Sci 2021;66:4191-4196.

34. Clough JN, Hill KL, Duff A, et al. Managing an IBD infusion unit during the COVID-19 pandemic: service modifications and the patient perspective. Inflamm Bowel Dis 2020;26:e125-e126.

35. Schlabitz F, Teich N, Riesner-Wehner A, et al. Inflammatory bowel disease and COVID-19: how have patients coped so far? J Clin Gastroenterol 2021 Feb 3 [Online ahead of print]. doi: 10.1097/ MCG.0000000000001504

36. Trindade IA, Ferreira NB. COVID-19 pandemic's effects on disease and psychological outcomes of people with inflammatory bowel disease in Portugal: a preliminary research. Inflamm Bowel Dis 2021;27:1224-1229.

37. Feitosa MR, Parra RS, de Camargo HP, et al. COVID-19 quarantine measures are associated with negative social impacts and compromised follow-up care in patients with inflammatory bowel disease in Brazil. Ann Gastroenterol 2021;34:39-45.

38. Te Groen M, Derks MEW, Kuijpers CCHJ, Nagtegaal ID, Hoentjen F. Reduction in inflammatory bowel disease healthcare during the coronavirus disease 2019 pandemic: a nationwide retrospective cohort study. Gastroenterology 2021;160:935-937.

39. Cheung KL, Ten Klooster PM, Smit C, de Vries H, Pieterse ME. The impact of non-response bias due to sampling in public health studies: A comparison of voluntary versus mandatory recruitment in a Dutch national survey on adolescent health. BMC Public Health 2017;17:276.

40. Effenberger M, Grabherr F, Mayr L, et al. Faecal calprotectin indicates intestinal inflammation in COVID-19. Gut 2020;69:1543-1544.

41. Mao R, Qiu Y, He JS, et al. Manifestations and prognosis of gastrointestinal and liver involvement in patients with COVID-19: a systematic review and meta-analysis. Lancet Gastroenterol Hepatol 2020;5:667-678.

42. D’Amico F, Danese S, Peyrin-Biroulet L. Systematic review on inflammatory bowel disease patients with coronavirus disease 2019: it is time to take stock. Clin Gastroenterol Hepatol 2020;18:2689-2700.

43. Jena A, Kumar-M P, Singh AK, Sharma V. Fecal calprotectin levels in COVID-19: lessons from a systematic review on its use in inflammatory bowel disease during the pandemic. Dig Liver Dis 2021;53:295-297.

44. Iacucci M, Cannatelli R, Labarile N, et al. Endoscopy in inflammatory bowel diseases during the COVID-19 pandemic and post-pandemic period. Lancet Gastroenterol Hepatol 2020;5:598-606.

45. Danese S, Sands B, Ng SC, Peyrin-Biroulet L. The day after COVID-19 in IBD: how to go back to 'normal'. Nat Rev Gastroenterol Hepatol 2020;17:441-443.

46. Chetcuti Zammit S, Sidhu R. Capsule endoscopy - recent developments and future directions. Expert Rev Gastroenterol Hepatol 2021;15:127-137.

47. Lopez A, Billioud V, Peyrin-Biroulet C, Peyrin-Biroulet L. Adherence to anti-TNF therapy in inflammatory bowel diseases: a systematic review. Inflamm Bowel Dis 2013;19:1528-1533.

48. Bots SJ, Kuin S, Ponsioen CY, et al. Relapse rates and predictors for relapse in a real-life cohort of IBD patients after discontinuation of anti-TNF therapy. Scand J Gastroenterol 2019;54:281-288.

49. Sud A, Jones ME, Broggio J, et al. Collateral damage: the impact on outcomes from cancer surgery of the COVID-19 pandemic. Ann Oncol 2020;31:1065-1074. 